\title{
Mitochondrial active Ras2 protein promotes apoptosis and regulated cell death in a cAMP/PKA pathway-dependent manner in budding yeast.
}

\author{
Barbara Bonomelli, Enzo Martegani * and Sonia Colombo *
}

\author{
Department of Biotechnology and Biosciences, University of Milano-Bicocca, Piazza della Scienza \\ 2, 20126 Milan, Italy \\ *Correspondence: Sonia Colombo, Department of Biotechnology and Biosciences, University of \\ Milano-Bicocca, Piazza della Scienza 2, 20126, Milan, Italy. Tel.: +390264483551; E-mail: \\ sonia.colombo@unimib.it; \\ Enzo Martegani, Department of Biotechnology and Biosciences, University of Milano-Bicocca, \\ Piazza della Scienza 2, 20126, Milan, Italy. Tel.: +390264483533; E-mail: enzo.martegani@unimib.it
}

\begin{abstract}
In previous papers, using the eGFP-RBD3 probe, which binds Ras-GTP with high affinity, we showed that activated Ras proteins are localized to the plasma membrane and in the nucleus in wild-type Saccharomyces cerevisiae cells growing exponentially on glucose, while an aberrant accumulation of activated Ras in mitochondria correlates to mitochondrial dysfunction, accumulation of ROS and an increase of apoptosis. In this paper, we show that lack of TPS1, which is known to trigger apoptosis in S. cerevisiae, induces localization of active Ras proteins in mitochondria, confirming the above-mentioned correlation. Next, by characterizing the ras $1 \Delta$ and ras $2 \Delta$ mutants concerning localization of active Ras proteins and propensity to undergo cell death, we show that active Ras2 proteins, which accumulate in the mitochondria following addition of acetic acid, a well-known pro-apoptotic stimulus, might be the GTPases involved in regulated cell death, while active Ras1 proteins, constitutively localized in mitochondria, might be involved in a pro-survival molecular machinery. Finally, by characterizing the gpa $2 \Delta$ and cyr1 $\Delta$ mutants concerning the propensity to undergo cell death, we show that active mitochondrial Ras proteins promote apoptosis through the cAMP/PKA pathway.
\end{abstract}

Keywords: Acetic acid; Mitochondria; ROS; Apoptosis; Regulated cell death; Ras/cAMP/PKA pathway; Yeast; S. cerevisiae

\section{Introduction}

The regulated cell death (RCD) includes a set of mechanisms aimed at the targeted elimination of superfluous, irreversibly damaged and/or potentially harmful cells, with an advantage for the body's homeostasis. RCD, as well as autophagy, can be activated in response to cellular stress. Dysfunctions in the regulation of RCD underlie several human diseases [1]. RCD is evolutionarily conserved among mammals and unicellular eukaryotes, such as the yeast Saccharomyces cerevisiae, which is one of the model organisms used to study apoptosis, a form of regulated cell death $[2,3]$. Several yeast genes involved in apoptosis have been identified, presenting an ortholog in mammals. These include genes encoding a caspase (Yca1), apoptosis inducing factor (Aif1), OMI serine protease (Nma111) and endonuclease G (Nuc1) [4-8]. The apoptotic pathway can be activated by the expression of pro-apoptotic heterologous proteins such as Bax [9], but also by exogenous agents such as hydrogen peroxide and acetic acid $[10,11]$. In particular acetic acid has been widely used as 
inducer of apoptosis in yeast [5, 11], since according to Sokolov et al [12] it triggers a ROS (Reactive Oxygen Species) dependent death.

The Saccharomyces cerevisiae contains two RAS genes, RAS1 and RAS2 coding for two proteins showing a significant homology to the mammalian Ras proteins [13, 14]. Yeast Ras1 and Ras2 proteins, like their mammalian counterparts, undergo extensive posttranslational modification (farnesylation, palmitoylation and carboxymethylation) and are deposited on the inner surface of the plasma membrane by a specialized transport mechanism [15-17]. The Ras proteins belong to a superfamily of small GTPases that cycle between an inactive state, when loaded with GDP, and an active state, when loaded to GTP. In budding yeast, the Ras proteins activity is tuned by two classes of regulatory proteins: Guanine nucleotide Exchange Factors (GEFs) Cdc25 and Sdc25, which stimulate the GDP/GTP exchange and Ira1 and Ira2 GTPase Activating Proteins (GAPs), which stimulate the intrinsically low Ras GTPase activity [18]. The molecular mechanisms of modulation of Ras proteins activity have been conserved during evolution, as it was confirmed by the functional interchangeability of Ras proteins and their regulators in mammals and budding yeast [18, 19].

In previous papers [20-22] we give evidences that in budding yeast exists a correlation between mitochondrial localization of Ras-GTP and apoptosis. In particular, we demonstrated that deletion of WHI2, a gene coding for a protein known to influence cell cycle exit under conditions of nutritional stress, leads to the loss of coordination between nutritional sensing and actin regulation, resulting in the failure to correctly traffic Ras 2 to the vacuole. Consequently, Ras2 protein localizes to the mitochondrial surface in its active form. This leads to a failure to shut down Ras signalling, to mitochondrial dysfunction, accumulation of damaging ROS and cell death [20] In a subsequent work, we showed that addition of acetic acid to $S$. cerevisiae wild type cells causes within five minutes a delocalization of active Ras from plasma membrane and nucleus to mitochondria [21]. Furthermore, we demonstrated that addition of either acetic acid or hydrogen peroxide to $h x k 2 \Delta$ cells, showing a constitutive localization of active Ras at the mitochondria, causes an increase in the level of ROS, mitochondrial dysfunctions and an increase of both apoptotic and necrotic cells compared with the wild-type strain [21, 22]. Finally, we showed that also lack of SNF1, the homolog of the AMP-activated protein kinase (AMPK) in S. cerevisiae, induces localization of active Ras in mitochondria and triggers apoptosis in this microorganism [23].

The two Ras proteins of S.cerevisiae, Ras1 and Ras2 cannot be discriminated by the eGFPRBD3 probe we used to investigate the localization of active Ras proteins (Ras-GTP) [24]. Consequently, in order to explore which one of these two GTPases is actually involved in apoptosis and cell death, we characterized the ras $1 \Delta$ and ras $2 \Delta$ mutants concerning localization of active Ras proteins and propensity to undergo these cellular processes, following a pro-apoptotic stimulus. We show that the Ras2 protein might be the GTPase involved in apoptosis and cell death induced by addition of acetic acid, while active Ras1 protein is localized in mitochondria and might be involved in a pro-survival molecular machinery. Next, we aimed to investigate whether the accumulation of active Ras proteins in the mitochondria determines an increase in the level of ROS, apoptosis and cell death in a cAMP/PKA pathway dependent manner $[25,26]$, since data reported in literature show that many proteins of the Ras/cAMP pathway, including Ira2, Cyr1, Bcy1 and Tpk1 are 
located in the mitochondria [27,28], suggesting that Ras-dependent transduction pathways could also originate and/or be "managed" by these organelles. Our results show that indeed active mitochondrial Ras2 protein promotes apoptosis and cell death through the cAMP/PKA pathway. Finally, we show that also in the $t p s 1 \Delta$ mutant $[29,30]$ a correlation exists between aberrant accumulation of activated Ras in mitochondria and propensity of the cells to undergo apoptosis.

\section{Materials and Methods}

2.1. Yeast strains, plasmids and media

The yeast strains used in this study are indicated in Table 1.

Table1. List of yeast strain

\begin{tabular}{|c|c|c|}
\hline Strain & Main Genotype & Source/reference \\
\hline W303-1A & MATa ade2-1 can1-100 his3-11,15 leu2-3112 trp1-1 ura3-1 & $\begin{array}{l}\text { Thomas and Rothstein, } 1989 \\
\text { [31] }\end{array}$ \\
\hline YSH290 & W303-1A with tps $1: \because T R P 1$ & Hohmann et al., 1993 [32] \\
\hline SP1 & MATa his3 leu 2 ura3 trpl ade8 canl & Nikawa et al. [33] \\
\hline ST-1 & SP1with $\operatorname{ras} 1:: U R A 3$ & $\begin{array}{l}\text { kindly provided by J. Thevelein } \\
\text { KU Leuven [34] }\end{array}$ \\
\hline KP-2 & SP1with $\operatorname{ras} 2:: U R A 3$ & $\begin{array}{l}\text { kindly provided by J.Thevelein, } \\
\text { KU Leuven [34] }\end{array}$ \\
\hline SC591 & W303-1A with ras2::URA3 & Our laboratory \\
\hline W303-1A gpa2d & W303-1A with gpa2::LEU2 & $\begin{array}{l}\text { kindly provided by } \mathrm{J} . \\
\text { Winderickx,,KU Leuven }\end{array}$ \\
\hline GG104 & $\begin{array}{c}\text { W303-1A with } p d e 2:: \text { TRP } 1, \text { cyrl }:: \operatorname{KanMX} 2, \text { msn } 2:: H I S 3, \\
\text { msn } 4:: T R P 1\end{array}$ & Roosen et al., (2005) [35] \\
\hline
\end{tabular}

The plasmid pYX212-eGFP-RBD3 encoding for the eGFP-RBD3 probe [24] was used to localize Ras-GTP in yeast cells through fluorescence microscopy analyses. Deletion of RAS2 in the W303-1A strain was made using polymerase chain reaction (PCR)-based strategy as previously described [36]. In particular, a PCR-cassette containing URA3 amplified from plasmid YCp50 was used. All the primers used in this study are listed in Supplementary Table I. Yeast cells were transformed according to Gietz et al. [37]. Rich medium contained $2 \% \mathrm{w} \mathrm{v}^{-1}$ glucose, $2 \% \mathrm{w} \mathrm{v}^{-1}$ peptone, $1 \% \mathrm{w} \mathrm{v}^{-1}$ yeast extract (YPD). Synthetic complete media (SD) contained either $2 \%$ glucose or $2 \%$ galactose, $6.7 \mathrm{~g} / 1 \mathrm{YNB}$ w/o amino acids (supplied by ForMedium $^{\mathrm{TM}}$, United Kingdom) and the proper selective drop-out CSM (Complete Synthetic Medium, supplied by ForMedium ${ }^{\mathrm{TM}}$, United Kingdom). Culture density was measured with a Coulter Counter (Coulter mod. Z2) on mildly sonicated, diluted samples and we monitored the growth and defined the exponential phase by measuring the cell number $/ \mathrm{ml}$ at discrete interval $(30 \mathrm{~min})$ and plotting the log of cell concentration as a function of time. YEPD plates contained $2 \% \mathrm{w} \mathrm{v}^{-1}$ glucose, $2 \% \mathrm{w} \mathrm{v}^{-1}$ peptone, $1 \% \mathrm{w} \mathrm{v}^{-1}$ yeast extract and $2 \% \mathrm{w} \mathrm{v}^{-1}$ agar.

\subsection{Acetic acid treatment}


Cells were grown at $30^{\circ} \mathrm{C}$ to exponential-phase (in the range $0.6-1 \times 10^{7}$ cells $\mathrm{mL}^{-1}$ ) in SD medium, harvested, resuspended (on average $10^{7}$ cells $\mathrm{mL}^{-1}$ ) in fresh SD medium adjusted to $\mathrm{pH} 3.0$ (set with $\mathrm{HCl}$ ) and treated with acetic acid (Riedel-deHaeden) at the indicated concentration. Cells were incubated for $200 \mathrm{~min}$ at $30^{\circ} \mathrm{C}$ with shaking (160 rpm) [21].

\subsection{Viability assay}

Cells were grown in SD medium at $30^{\circ} \mathrm{C}$ until exponential phase (in the range $0.6-1 \times 10^{7}$ cells $\mathrm{mL}^{-1}$ ) and treated with $80 \mathrm{mM}$ acetic acid for $200 \mathrm{~min}$. Cell number was measured before and after acetic acid treatment using a Coulter Counter (Coulter mod. Z2) and 1000 cells were plated on YEPD agar plates in triplicate. Viability was determined by measuring colony forming units (cfu) after 3 days of growth at $30^{\circ} \mathrm{C}$ [21].

\subsection{Dihydrorhodamine 123 (DHR123) staining}

ROS were detected with DHR123 (Sigma Aldrich) essentially as described by Madeo et al. $[10,21]$. Cells were grown in SD medium at $30^{\circ} \mathrm{C}$ until exponential phase (in the range 0.6 $x 10^{7}$ cells $\mathrm{mL}^{-1}$ ) and treated with acetic acid as described above for $80 \mathrm{~min}$. Then, DHR123 was added directly to the culture medium at the final concentration of $5 \mu \mathrm{g} \mathrm{mL} \mathrm{m}^{-1}$ (from a 2.5 $\mu \mathrm{g} \mu \mathrm{L}^{-1}$ stock solution) and incubation at $30^{\circ} \mathrm{C}$ with shaking was prolonged for additional $120 \mathrm{~min}$ in the dark at $30^{\circ} \mathrm{C}$. After a total of $200 \mathrm{~min}$ incubation, cells were diluted in $50 \mathrm{mM}$ filtered TrisHCl pH 7.5 to $10^{6} \mathrm{~mL}^{-1}$ and analysed using a cytofluorimeter (CytoflexS, Beckman) with excitation and emission wavelength of 488 and $525 \mathrm{~nm}$ respectively. A total of 20.000 events were acquired for each sample and data were processed using CytExpert software.

\subsection{Annexin $V$ and propidium iodide (PI) staining}

(FITC)-conjugated recombinant Annexin V (Immuno Tools) was used for the detection of phosphatidylserine exposed in the membrane of apoptotic cells as reported previously [2123]. Cells were harvested after $200 \mathrm{~min}$ of acetic acid treatment, washed with sorbitol buffer (1M sorbitol, 0.1M NaH2PO4, pH 8.0) and the cell wall was digested with Zymolyase 20T (Seikagaku Biobusiness Corporation) for about $35 \mathrm{~min}$ at $37^{\circ} \mathrm{C}$. Cells were then washed two times with binding buffer (10 mM Hepes/NaOH pH 7.4, $140 \mathrm{mM} \mathrm{NaCl}, 2.5 \mathrm{mM} \mathrm{CaCl2,} 1.2$ $\mathrm{M}$ sorbitol). Spheroplasts were resuspended in $35 \mu \mathrm{L}$ of binding buffer and incubated with $2.5 \mu \mathrm{L}$ of Annexin V (ImmunoTools) and $2 \mu \mathrm{L}$ of a PI (Fluka) working solution (50 $\mu \mathrm{g} \mathrm{mL}^{-1}$ in $10 \mathrm{mM}$ TrisHCl pH 7.0) for $15 \mathrm{~min}$ in the dark at room temperature. After staining, the samples were resuspended in binding buffer and analysed using a cytofluorimeter (CytoflexS, Beckman) with excitation at $488 \mathrm{~nm}$ and $525 \mathrm{~nm}$ emission for Annexin and 585 $\mathrm{nm}$ emission for PI. A total of 30.000 events were acquired for each sample and data were processed using CyExpert software.

\subsection{Fluorescence microscopy}

Cells expressing the eGFP-RBD3 probe [24] were grown in SD medium at $30^{\circ} \mathrm{C}$ until exponential phase(in the range $0.6-1 \times 10^{7}$ cells $\mathrm{mL}^{-1}$ ) and incubated with the mitochondrial marker rhodamine B hexyl ester perchlorate (Molecular Probes, Eugene, OR) $100 \mathrm{nM}$ final 
concentration for about 5 min before imaging. Images were acquired with a Nikon Eclipse $90 \mathrm{i}$ microscope equipped with a 60X oil immersion objective and a standard FITC filter set for GFP fluorescence. Images were recorded digitally using a Nikon DS-Qi1Mc camera and processed using Adobe Photoshop (Adobe Systems, Inc.) and ImageJ software (https://imagej.nih.gov). Determination of the spatio-temporal localization of Ras-GTP after addition of glucose to tps $1 \Delta$ cells growing exponentially on $2 \%$ galactose medium was performed as following. Cells were grown in medium containing $2 \%$ galactose at $30^{\circ} \mathrm{C}$ till exponential phase. Subsequently, $100 \mu$ of cells were seeded on concanavalin A-coated cover glass for $10 \mathrm{~min}$. The cover glass was washed four times using $1 \mathrm{ml}$ of $2 \%$ galactose medium and put on top of a Thoma chamber. Images were acquired before and after addition of glucose with a Nikon Eclipse 90i microscope equipped with a 60X oil immersion objective and a standard FITC filter set for GFP fluorescence. Glucose was added by pipetting, between the cover glass and the Thoma Chamber, $45 \mu \mathrm{l}$ of $4 \%$ glucose medium. To avoid bleaching, the fluorescence images were acquired for $4 \mathrm{~s}$ every 1 minute for $10 \mathrm{~min}$ and the shutter was kept off in the meantime. The time series of images was then processed using ImageJ software.

\subsection{Statistical analysis}

All the experiments were done at least in triplicate and the mean and Standard deviation was shown. The Student's t test was used for assessing the significance of the experimental data. The experimental data were elaborated with Excel TM.

\section{Results and discussion}

\subsection{Lack of TPS1 gene induces localization of active Ras in mitochondria in budding yeast}

In the yeast Saccharomyces cerevisiae, trehalose-6P-synthase (Tps1) is involved in trehalose synthesis, a highly stable disaccharide, implicated in many cellular processes and especially known for its protective role in response to a variety of environmental and nutritional stresses [38]. In particular, Tps1 first produces trehalose-6-phosphate (T6P) from glucose-6P and UDP-Glucose, which is then dephosphorylated by a specific trehalose-6P-phosphatase (Tps2p), to yield to trehalose. Recent data published in literature show a new function for the Tps1 protein as a pro-survival factor during growth and apoptotic stress $[39,40]$. In particular Peeters et al [40] showed that addition of glucose to tps1 $\Delta$ cells grown on galactose triggers activation of Ras proteins and apoptosis. In previous papers, we show that activated Ras proteins (Ras-GTP) are localized to the plasma membrane and in the nucleus in wildtype yeast cells growing exponentially on glucose [24], while an aberrant accumulation of activated Ras in mitochondria correlates to mitochondrial dysfunction, accumulation of ROS and an increased sensitivity to pro-apoptotic treatments [20-23]. Therefore, in this paper we investigated the localization of active Ras proteins in the $t p s 1 \Delta$ strain using the eGFP-RBD3 probe [24] in order to verify whether also in this mutant a correlation exists between aberrant accumulation of activated Ras in mitochondria and apoptosis. Due to the inability of the tps1 $\Delta$ mutant to grow on glucose $[29,32]$, we performed the experiment during growth on galactose medium. As a control, the localization of active Ras proteins was also investigated in W303-1A cells during growth on the same medium. As expected 
and in agreement with previously published data [24], in W303-1A cells growing on medium containing galactose, active Ras proteins were partially localized to the plasma membrane, in the nucleus and in mitochondria. As shown in Figure 1, in tps $1 \Delta$ cells active Ras proteins were almost totally localized in mitochondria (90\%), indicating that also in this mutant a correlation exists between aberrant accumulation of activated Ras in mitochondria and propensity of the cells to undergo apoptosis. Moreover, we show that glucose addition to galactose-grown tps $1 \Delta$ cells triggered a fast and marked increase of green fluorescence in mitochondria, suggesting that there is an increase of active Ras in these organelles (Figure 2 ). This result agrees with published data showing that glucose addition to galactose-grown tps1 $\Delta$ cells triggers a rapid and dramatic activation of Ras [40].

A
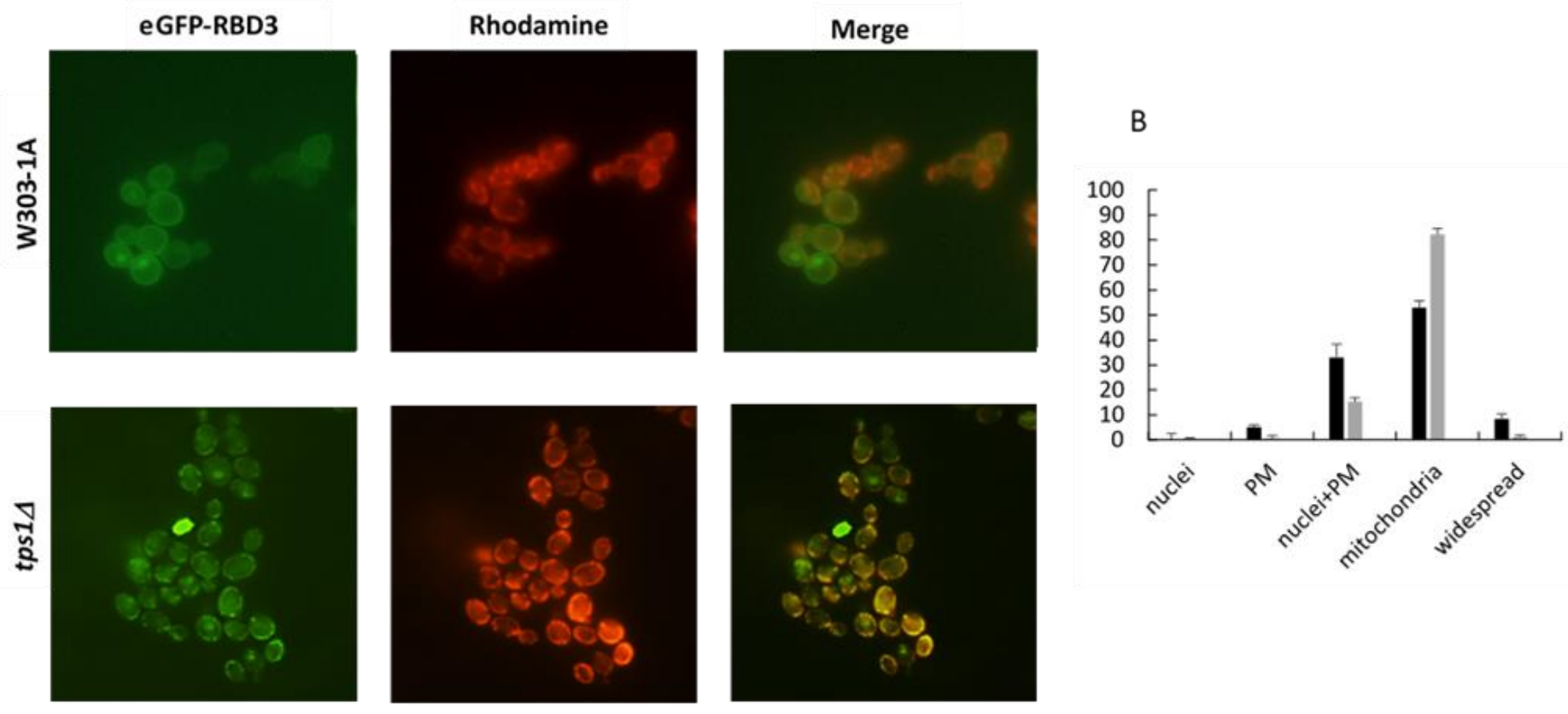

Figure 1. Localization of active Ras proteins in the tps1 $1 \Delta$ mutant. (A) Localization of eGFP fluorescence and redfluorescent rhodamine $\mathrm{B}$ hexyl ester in W303-1A and tps1 $\Delta$ cells expressing the eGFP-RBD3 probe and growing exponentially on galactose medium. (B) Subcellular distribution of eGFP fluorescence in W303-1A (black) and $\operatorname{tps} 1 \Delta$ (grey) cells. Cells belonging to each class were counted and expressed as a percentage on the total number of fluorescent cells. Approximately 300 cells were scored and error bars represent the standard deviation. 
Fig. 2
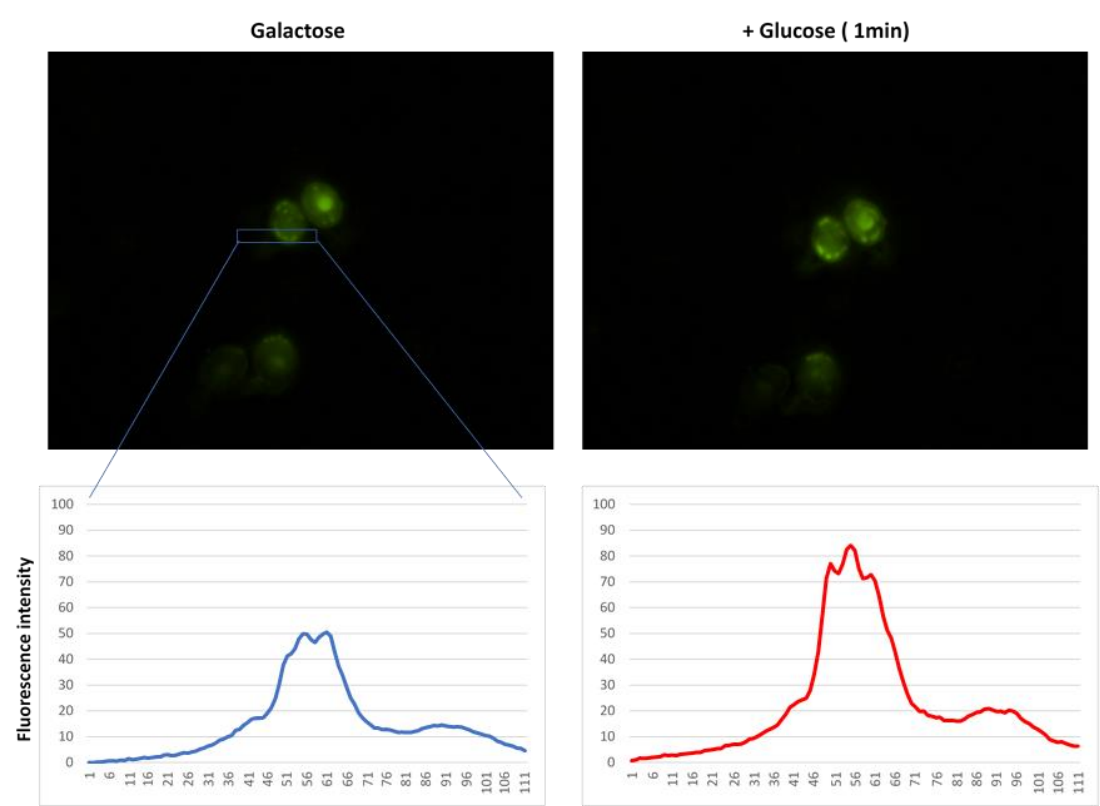

Figure 2. Glucose triggers a rapid and marked increase of fluorescence in mitochondria of tps1 $1 \Delta$ cells. eGFP fluorescence level before and $1 \mathrm{~min}$ after addition of glucose to tps1 $\Delta$ cells expressing the eGFP-RBD3 probe and growing on galactose medium. Cells growing in $2 \%$ galactose medium were seeded on concanavalin A-coated cover glass and mounted on top of a Thoma chamber (see Materials and Methods for details). Images were acquired before and 1 minute after addition of glucose with a Nikon Eclipse 90i microscope equipped with a 60X oil immersion objective and with the same setting of exposure time and imaging (4s, 1.2 gain) (upper). Images was than analysed with ImageJ software to obtain the pixel values (profile) (bottom) along the rectangular ROI overimposed in blue in the figure.

\subsection{The Ras2 protein is the GTPase involved in apoptosis induced by addition of acetic acid}

S. cerevisiae has two Ras proteins, Ras1 and Ras2 [13]. Since the eGFP-RBD3 probe is unable to discriminate between them, in order to investigate which one of the two proteins was involved in apoptosis, we characterized the ras $1 \Delta$ and ras $2 \Delta$ mutants concerning localization of active Ras proteins and propensity to undergo apoptosis and cell death. Our results show that in glucose growing cells, deletion of RAS2 caused a mitochondrial localization of the probe, while deletion of RAS1 did not impair the nuclear and plasma membrane localization of active Ras2 (Figure 3). We also investigated the localization of active Ras2 protein after addition of $40 \mathrm{mM}$ acetic acid, a well-known inducer of apoptosis in yeast, to ras $1 \Delta$ cells. We could show that in this strain, active Ras2 protein moved from plasma membrane and nuclei to the mitochondria within 5 minutes, suggesting that active Ras2 proteins might actually be the GTPases involved in apoptosis and cell death (Figure 3B). However, a possible contribution of active Ras1 protein in this cellular process might not be excluded. In order to investigate the role of active Ras1 proteins in apoptosis and cell death, we conducted cell survival experiments, assessed oxidative damage (ROS level) and the expression of cytological markers of apoptosis and necrosis, in the presence and absence of acid acetic, in the ras $2 \Delta$ strain. 
$\operatorname{ras} 2 \Delta$
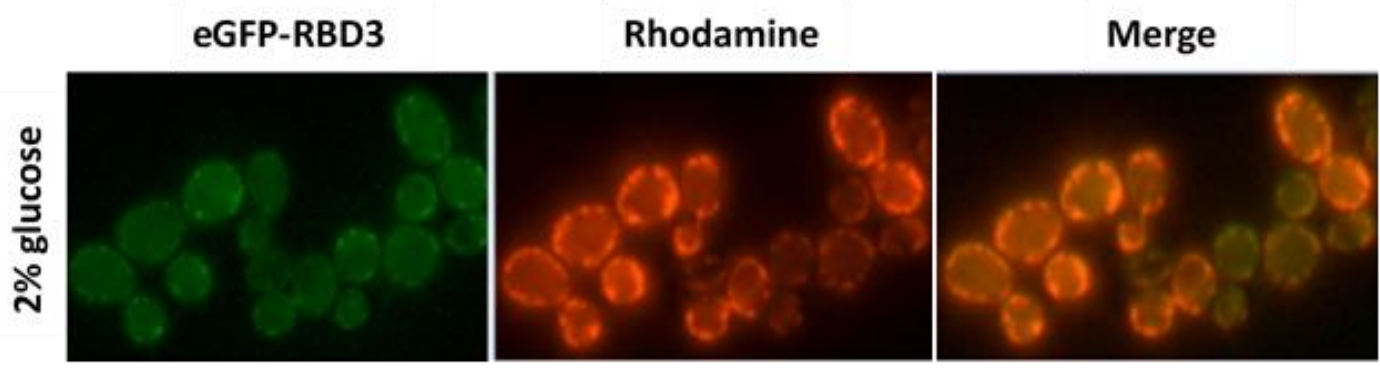

B

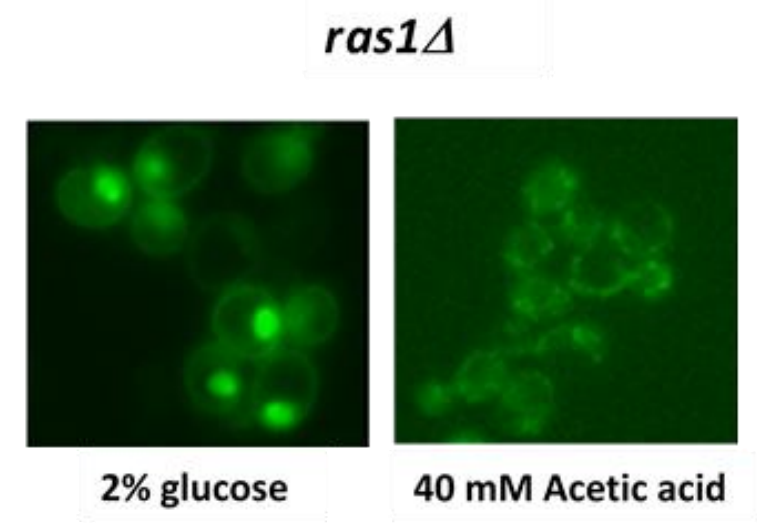

Figure 3. Localization of active Ras proteins in RAS deletion mutants. (A) Localization of eGFP fluorescence and red-fluorescent rhodamine B hexyl ester in SP1 ras2 $\Delta$ cells expressing the eGFP-RBD3 probe and growing exponentially on glucose medium. (B) Localization of eGFP fluorescence in SP1 ras1 $\Delta$ cells expressng the eGFP-RBD3 probe, growing exponentially on glucose medium and 5 minutes after addition of $40 \mathrm{mM}$ acetic acid.

After treatment with $80 \mathrm{mM}$ acetic acid for 200 minutes at $30^{\circ} \mathrm{C}$, ras $2 \Delta$ cells showed a significant increase in cell survival when compared with wild-type cells (Figure 4A). Congruently, the amount of ROS was significantly reduced in ras $2 \Delta$ cells compared to wildtype cells (Figure 4B, Supplementary Figure 2). We also measured the level of cytological markers of apoptosis and necrosis in the ras $2 \Delta$ mutant by flow-cytometry. In particular, combined Annexin V/propidium iodide (PI) staining was used to discriminate between early apoptotic (Annexin V+/PI-), late apoptotic/secondary necrotic (Annexin V+/PI+) and primary necrotic (Annexin $\mathrm{V}-/ \mathrm{PI}+$ ) deaths. Our results showed that the percentage of ras $2 \Delta$ cells in late apoptosis/secondary necrosis following treatment with $80 \mathrm{mM}$ acetic acid was significantly reduced compared to that obtained for the corresponding wild strain (Figure 4C (c), Supplementary Figure 3), while the percentage of primary necrotic cells was very low, both in ras $2 \Delta$ and wild type strains (Figure $4 \mathrm{C}$ (b)). In summary, data on cell survival, ROS level and percentage of cells in late apoptosis/secondary necrosis are consistent and indicate that constitutively mitochondrial localized active Ras1 protein is not related to the 
propensity of the cells to undergo a regulated cell death modality with characteristic features of apoptosis, following addition of acetic acid. However, unexpectedly, the percentage of cells in early apoptosis in the ras $2 \Delta$ strain, following treatment with acetic acid, was significantly high compared with that obtained for the wild strain (Figure 4C (a)). This data, which is apparently in contrast with the above data, might indicate that in yeast, as well as in higher eukaryotes, apoptosis is divided into phases, being the initial phase (early apoptosis) reversible and secondary necrosis (combined necrotic and apoptotic features) the final stage of the apoptotic process [3]. Moreover, the presented results are congruent with recent data demonstrating that late apoptosis/secondary necrosis might not be necessarily an accidental consequence of early apoptosis, but a finely regulated process controlled by a specific molecular machinery [41] and that secondary necrotic cells might actually undergo secondary necrosis following other cell death subroutines [3]. In conclusion, taken together our data suggest that: 1) active Ras2 proteins, which accumulate in the mitochondria following a pro-apoptotic stimulus (acetic acid for instance), might be the GTPases involved in apoptosis and cell death; 2) mitochondrial active Ras1 proteins might be involved in a pro-survival molecular machinery. The two proteins Ras1 and Ras2 have similar and overlapping function in the activation of adenylate cyclase [42, 43], but they have different expression at the level of mRNA and protein [44]. In addition, Ras2 is much more abundant than Ras1 [44, 45] (20000 mol/cell versus 2000 of Ras1 [45]); moreover, it has been shown that these two proteins have an opposite function also in replicative aging of yeast cells [46] and in stress response [47].

Fig. 4

A

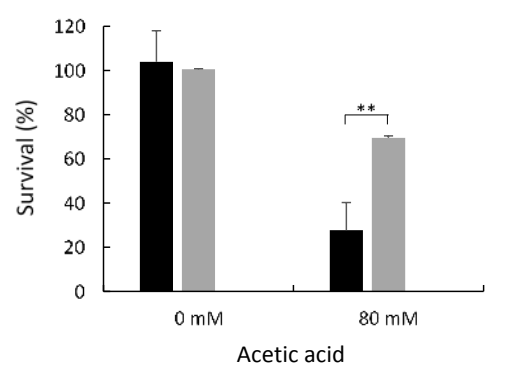

B

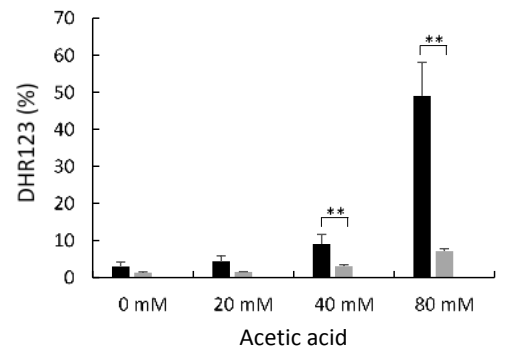

C
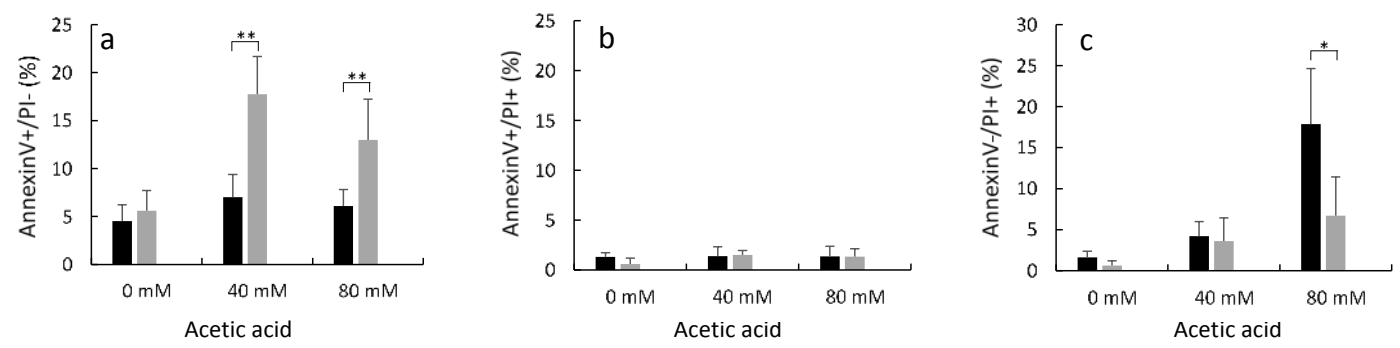

Figure 4. Cell survival, ROS accumulation and cell death in wild-type and ras $2 \Delta$ cells upon acetic acid treatment. (A) Cell survival of W303-1A and W303-1A ras2 $\Delta$ cells. Cell viability of W303-1A (black bars) and W303-1A ras2 $\Delta$ 
(grey bars) untreated cells or treated with $80 \mathrm{mM}$ acetic acid was analyzed by measuring colony-forming units (cfu) after 3 days of growth at $30^{\circ} \mathrm{C}$. Cell survival is expressed as $\%$ to the cfu at time zero. (B) ROS accumulation in W303-1A and W303-1A ras2 $\Delta$ cells. W303-1A (black bars) and W303-1A ras2 $\Delta$ (grey bars) exponentially growing cells were treated with either 20,40 or $80 \mathrm{mM}$ acetic acid for 200 minutes at $30^{\circ} \mathrm{C}$ with shaking (160rpm). Dihydrorhodamine 123 (DHR123) was used to assay ROS accumulation. The means of three independent experiments with standard deviations are reported. Student's t-test $* \mathrm{P}<0.05$ and $* * \mathrm{P}<0.01$. (C) Assessment of cell death by FITC-coupled annexinV and PI staining. W303-1A (black bars) and W303-1A ras $2 \Delta$ (grey bars) cells growing exponentially on glucose medium were treated with 40 or $80 \mathrm{mM}$ acetic acid for 200 minutes at $30^{\circ} \mathrm{C}$, before being processed for determination of phosphatidylserine externalization and membrane integrity by flow cytometry. 30000 events have been evaluated. The means of 3 independent experiments with standard deviations are reported. Student's $t$-test $* P<0.05$ and $* * P<0.01$.

\subsection{Active mitochondrial Ras2 protein promote apoptosis and cell death through the cAMP/PKA} pathway

Data in literature show that some components of the yeast cAMP/PKA pathway, such as Ira2, adenylate cyclase (Cyr1) [27], and catalytic (Tpk1) and regulatory (Bcy1) subunits of Protein Kinase A (PKA) [28], are also significantly present on mitochondria. Consequently, we investigated whether active mitochondrial Ras2 proteins promote apoptosis and cell death in a cAMP/PKA pathway-dependent manner, since it is well known that Ras proteins in budding yeast activate adenylate cyclase and PKA $[15,18,34]$. To this aim, we measured cell survival and the levels of ROS and cytological markers of apoptosis and necrosis, in the presence and absence of acid acetic, in two yeast mutants that showed a downregulation of the pathway and reduced activation of PKA: the gpa $2 \Delta$ and cyr $1 \Delta$ strains. In yeast, Gpa2 is a G-protein that activates adenylate cyclase in response to glucose [48]. In the gpa2॰ mutant the cAMP/PKA pathway is downregulated and active Ras proteins are solely localized in the mitochondria [24], while in the cyr1@ strain adenylate cyclase is lacking [35], the cAMP/PKA pathway is totally compromised and a consistent percentage (about 30\%) of active Ras proteins is localized in these cell organelles [24]. After treatment with $80 \mathrm{mM}$ acetic acid for 200 minutes at $30^{\circ} \mathrm{C}$, both gpa $\Delta \Delta$ and cyr $1 \Delta$ cells showed a significant increase in cell survival (around 90\%) when compared with wild-type cells (Figure 5A). Congruently, the percentage of ROS was significantly reduced in these mutants compared to wild-type cells (Figure 5B). Finally, we measured the level of cytological markers of apoptosis and necrosis in the gpa $2 \Delta$ and cyr $1 \Delta$ mutants. The percentage of gpa $2 \Delta$ and cyr $1 \Delta$ cells in late apoptosis/secondary necrosis following treatment with $80 \mathrm{mM}$ acetic acid was significantly reduced compared to that obtained for the corresponding wild type strain (Figure 4C (c)). As previously shown for the ras $2 \Delta$ strain, also for the gpa $2 \Delta$ and cyr $1 \Delta$ mutants, the percentage of cells in early apoptosis (Annexin V+/PI-), following treatment with $40 \mathrm{mM}$ and $80 \mathrm{mM}$ acetic acid, was significantly high compared with that obtained for the wild strain (Figure 4C (a)). In summary, taken together, our data suggest that mitochondrial active Ras2 proteins promote apoptosis and cell death through the cAMP/PKA pathway. 
Fig. 5

A

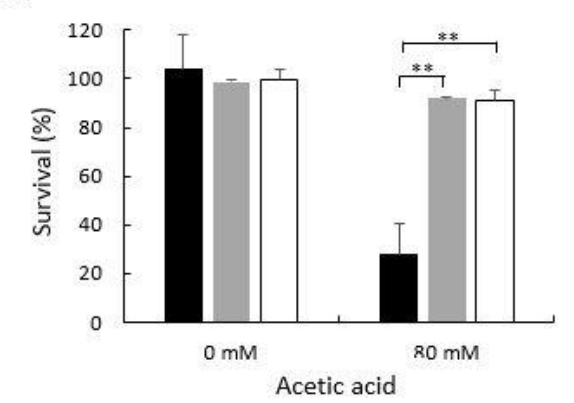

B

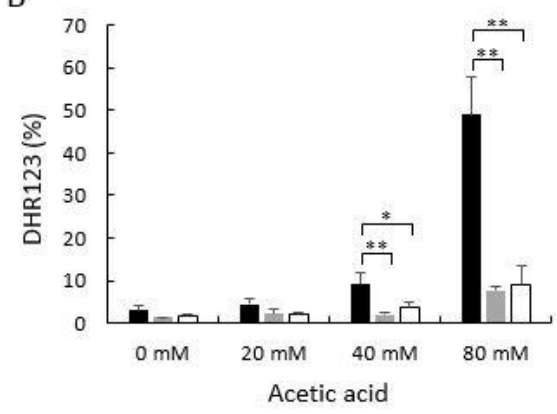

C
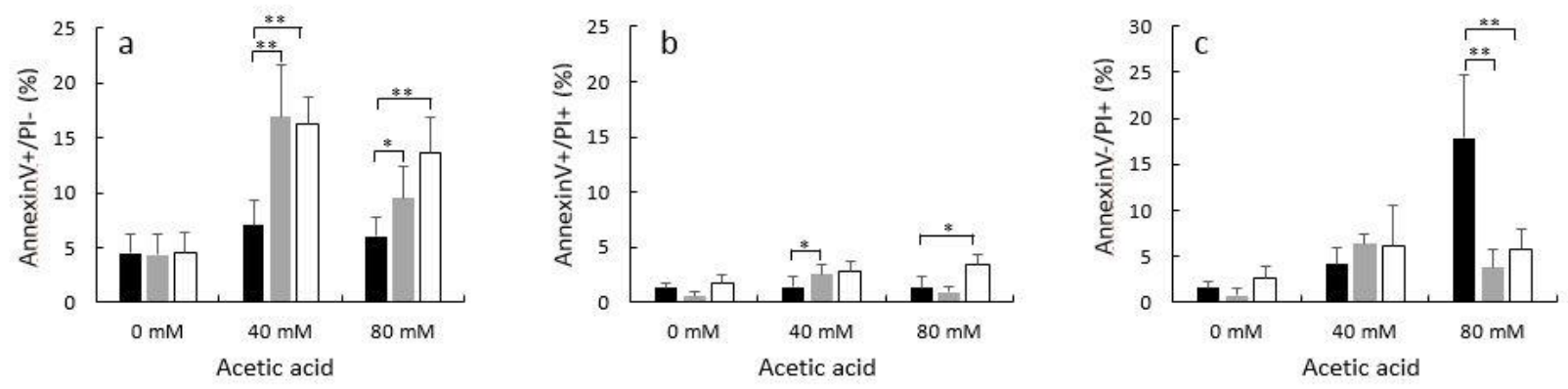

Figure 5. Cell survival, ROS accumulation and cell death in wild-type, gpa2 $\Delta$ and cyr1 $\Delta$ cells upon acetic acid treatment. (A) Cell survival of W303-1A, gpa2 $\Delta$ and cyr1 $\Delta$ strains. Cell viability of W303-1A (black bars), gpa2 $\Delta$ (grey bars) and cyr1 1 (white bars) untreated cells or treated with $80 \mathrm{mM}$ acetic acid was analyzed by measuring colony-forming units (cfu) after 3 days of growth at $30^{\circ} \mathrm{C}$. Cell survival is expressed as $\%$ to the cfu at time zero. (B) ROS accumulation in W303-1A, gpa2 $\Delta$ and cyr1 $\Delta$ cells. W303-1A (black bars), gpa2 $\Delta$ (grey bars) and cyr1 $\Delta$ (white bars) exponentially growing cells were treated with either 20, 40 or $80 \mathrm{mM}$ acetic acid for 200 minutes at $30^{\circ} \mathrm{C}$ with shaking (160rpm). Dihydrorhodamine 123 (DHR123) was used to assay ROS accumulation. The means of three independent experiments with standard deviations are reported. Student's t-test $* \mathrm{P}<0.05$ and $* * \mathrm{P}<0.01$. (C) Assessment of cell death by FITC-coupled annexinV and PI staining. W303-1A (black bars), gpa2 $\Delta$ (grey bars) and cyr1 1 (white bars) cells growing exponentially on glucose medium were treated with 40 or 80 $\mathrm{mM}$ acetic acid for 200 minutes at $30^{\circ} \mathrm{C}$, before being processed for determination of phosphatidylserine externalization and membrane integrity by flow cytometry. 30000 events have been evaluated. The means of 3 independent experiments with standard deviations are reported. Student's $t$-test $* P<0.05$ and $* * P<0.01$.

\section{Conclusion}

We previously showed that a correlation exists between mitochondrial localization of RasGTP and apoptosis. We did that using the eGFP-RBD3 probe, which binds activated Ras proteins with high affinity, but cannot discriminate between Ras1 and Ras2, the two Ras proteins of S. cerevisiae. Consequently, in this paper we explored which one of these two GTPases is actually involved in apoptosis and cell death by characterizing the ras $1 \Delta$ and ras $2 \Delta$ mutants concerning localization of active Ras proteins and propensity to undergo these cellular processes, following a pro-apoptotic stimulus. We could show that the Ras2 protein is actually the small $\mathrm{G}$ protein, which promotes apoptosis and cell death, and it does that through the cAMP/PKA pathway, while the Ras1 protein might be involved in a pro- 
survival process. We also demonstrate that lack of TPS1, which is known to trigger apoptosis in S. cerevisiae, induces localization of active Ras proteins in mitochondria, confirming the correlation between mitochondrial localization of Ras-GTP and apoptosis.

Funding: This work was supported by FAR - University of Milano Bicocca grants to S.C. and E.M.

Acknowledgments: We thank. P. van Dijck, KU Leuven, Belgium, for providing the tps1 $\Delta$ strain, J. Winderickx, KU Leuven, Belgium, for providing the W303-1A gpa2s strain and J. Thevelein, KU Leuven, Belgium, for providing ST-1 and KP-2 strains. We thank S. Citterio, Università Milano-Bicocca, for her precious technical support and N. Tosetto for technical help.

Conflicts of Interest: The authors declare no conflict of interest.

\section{References}

1. Tang, D.; Kang, R.; Vanden Berghe, T.; et al. The molecular machinery of regulated cell death Cell Research 2019, 29, 347-364.

2. Madeo, F.; Herker, E.; Wissing, S; et al. Apoptosis in yeast Curr Opin Microbiol 2004, 7, 655-60.

3. Carmona-Gutierrez, D.; Bauer, M.A.; Zimmermann, A.; et al. Guidelines and recommendations on yeast cell death nomenclature Microb. Cell 2018, 5 (1), 4-31.

4. Madeo, F.; Herker, E.; Maldener, C.; et al. A caspase-related protease regulates apoptosis in yeast Molecular Cell 2002, 9 (4), 911-917.

5. Guaragnella, N.; Pereira, C.; Sousa, M. J.; et al. YCA1 participates in the acetic acid induced yeast programmed cell death also in a manner unrelated to its caspase-like activity FEBS Letters 2006, 580 (30), 6880-6884.

6. Wissing, S.; Ludovico, P.; Herker; E.; et al. An AIF orthologue regulates apoptosis in yeast J. Cell Biol. 2004,166 (7), 969-974.

7. Fahrenkrog, B.; Sauder, U.; Aebi, U. The S. cerevisiae HtrA like protein Nma111p is a nuclear serine protease that mediates yeast apoptosis J. Cell Sci. 2004, 117 (1), 115-126.

8. Buttner, S.; Eisenberg, T.; Carmona-Gutierrez, D.; et al. Endonuclease G regulates budding yeast life and death Molecular Cell 2007, 25 (2), 233-246.

9. Ligr, M.; Madeo, F.; Fröhlich, E.; Hilt, W.; Fröhlich, K.-U.; Wolf, H.D. Mammalian Bax triggers apoptotic changes in yeast FEBS Letters 1998, 438 (1-2), 61-65.

10. Madeo, F.; Fröhlich, M. E.; Ligr, M.; et al. Oxygen stress: a regulator of apoptosis in yeast J Cell Biol, 1999, 145 (4), 757767.

11. Ludovico, P.; Sousa, M. J.; Silva, M. T.; Leão, C.; Côrte-Real, M. Saccharomyces cerevisiae commits to a programmed cell death process in response to acetic acid Microbiology 2001, 147 (9), 2409-2415.

12. Sokolov, S.; Knorre, D.; Smirnova, E. et al. Ysp2 mediates death of yeast induced by amiodarone or intracellular acidification Biochim Biophys Acta 2006, 1757, 1366-1370.

13. Powers,S.; Kataoka,T.; Fasano,O. et al. Genes in S. cerevisiae encoding proteins with domains homologous to the mammalian ras proteins Cell 1984, 36, 607-612.

14. Tamanoi,F., Walsh,M.; Kataoka,T.; Wigler, M. A product of yeast RAS2 gene is a guanine nucleotide binding protein Proc. Natl.Acad Sci USA 1984, 81, 6924-6928.

15. Crechet,J.; Cool,R.; Jacquet,E.,Lallemand,J. Characterization of Saccharomyces cerevisiae Ras1p and chimaeric constructs of Ras proteins reveals the hypervariable region and farnesylation as critical elements in the adenylyl cyclase signaling pathway Biochemistry 2003, 42, 14903-14912.

16. Dong,X.; Mitchell,D.; Lobo,S. et al. Palmitoylation and plasma membrane localization of Ras2p by a nonclassical trafficking pathway in Saccharomyces cerevisiae Mol. Cell. Biol. 2003, 23, 6574- 6584.

17. Wang,G. ;Deschenes,R. Plasma membrane localization of Ras requires class C Vps proteins and functional mitochondria in Saccharomyces cerevisiae Mol. Cell. Biol. 2006, 26, 3243-3255.

18. Tisi,R.; Belotti,F.; Martegani, E. Yeast as a Model for Ras Signaling Methods in Molecular Biology 2014, Vol 1120, Cap 23.

19. Cazzanelli,G.; Pereira,F.; Alves,S. et al. The yeast Saccharomyces cerevisiae as a model for understanding RAS proteins and their role in human tumorigenesis Cells 2018,7,14. 
20. Leadsham, J.E.; Miller, K.; Ayscough, K.R.; et al. Whi2p links nutritional sensing to actin dependent Ras/cAMP/PKA regulation and apoptosis in yeast J. Cell Sci. 2009, 122, 706-715.

21. Amigoni, L.; Martegani, E.; Colombo, S. Lack of HXK2 induces localization of active Ras in mitochondria and triggers apoptosis in the yeast Sacharomyces cerevisiae Oxid. Med. Cell. Longev. 2013, 678473, https://doi.org/10.1155/2013/678473.

22. Amigoni, L.; Frigerio, G.; Martegani, E.; Colombo, S. Involvement of Aif1 in apoptosis triggered by lack of Hxk2 in the yeast Saccharomyces cerevisiae FEMS Yeast Res. 2016, 16 (3), https://doi.org/10.1093/femsyr/fow016 pii: fow016.

23. Bonomelli, B; Martegani, E; Colombo, S. Lack of SNF1 induces localization of active Ras in mitochondria and triggers apoptosis in the yeast Saccharomyces cerevisiae Biochem. Biophys. Res. Commun. 2020, 523, 130-134.

24. Broggi, S.; Martegani, E.; Colombo, S. Live-cell imaging of endogenous Ras-GTP shows predominant Ras activation at the plasma membrane and in the nucleus in Saccharomyces cerevisiae Int. J. Biochem. Cell Biol. 2013, 45, 384-394.

25. Burtner, C.R.; Murakami, C.J.; Kennedy, B.K.; Kaeberlein,M. A molecular mechanism of chronological aging in yeast Cell Cycle 2009, 8, 1256-1270.

26. Rego,A.; Mendes,F.; Costa,V.; Rodrigues Chaves,S.; Corte-Real, M. Pkh1p-Ypk1p and Pkh1p-Sch9p Pathways Are Activated by Acetic Acid to Induce a Mitochondrial-Dependent Regulated Cell Death Oxid.Med.Cell Longev. 2020, 7095078, https://doi.org/10.1155/2020/7095078.

27. Belotti, F.; Tisi, R.; Paiardi, C.; Rigamonti, M.; Groppi, S.; Martegani, E. Localization of Ras signaling complex in budding yeast Biochim. Biophys. Acta 2012, 1208-1216.

28. Galello, F.; Moreno, S.; Rossi S. Interacting proteins of protein kinase A regulatory subunit in Saccharomyces cerevisiae J. Proteomics 2014, 261-275.

29. Van Aelst, L. et al. Molecular cloning of a gene involved in glucose sensing in the yeast Saccharomyces cerevisiae. Mol. Microbiol. 1993, 8, 927-943.

30. Neves, M. J. et al. Control of glucose influx into glycolysis and pleiotropic effects studied in different isogenic sets of Sacharomyces cerevisiae mutants in trehalose biosynthesis. Curr. Genet. 1995, 27, 110-122.

31. Thomas, B.J.; Rothstein, R. Elevated recombination rates in transcriptionally active DNA Cell 1989, 56 (4) $619 \mathrm{e} 630$

32. Hohmann, S.; Neves, M.J.; de Koning, W.; Alijo, R.; Ramos, J.; Thevelein, J.M. The growth and signalling defects of the ggs1 (fdp1/byp1) deletion mutant on glucose are suppressed by a deletion of the gene encoding hexokinase PII Curr. Genet. 1993, 23, 281-289.

33. Nikawa, J.; Cameron, S.; Toda, T.; et al. Rigorous feedback control of cAMP levels in Saccharomyces cerevisiae Genes Dev. 1987, 1, 931-937.

34. Mbonyi, K.; Beullens, M.; Detremerie, K.; Geerts, L.; Thevelein J.M. Requirement of One Functional RAS Gene and Inability of an Oncogenic ras Variant To Mediate the Glucose-Induced Cyclic AMP Signal in the Yeast Saccharomyces cerevisiae Mol. Cell. Biol. 1988, 8 (8), 3051-3057.

35. Roosen, J.; Engelen, K.; Marchal, K.; Mathys, J.; Griffioen, G.; Cameroni, E.; et al. PKA and Sch9 control a molecular switch important for the proper adaptation to nutrient availability Mol. Microbiol. 2005, 55, 862-80.

36. Knop, M.; Siengers, K.; Pereira, G.; Zachariae, W.; Winson, B.; Nasmyth, K.; Schiebel, E. Epitope tagging of yeast genes using a PCR-based strategy: more tags and improved practical routines Yeast 1999, 15, 963-72.

37. Gietz, R.D.; Schiestl, R.H. Transforming yeasr with DNA Methods in Molecular and cellular Biology 1995, 5, 255-269.

38. Elbein, A.D.; Pan, Y.T.; Pastuszak, I.; Carroll, D. New insights on trehalose: amultifunctional molecule Glycobiology 2003, 13, 17-27.

39. Yoshiyama, Y.; Tanaka,K.; Yoshiyama,K.; Hibi, M.; Ogawa,J.; Shima,J. Trehalose accumulation enhances tolerance of Saccharomyces cerevisiae to acetic acid J. Biosc. Bioeng 2015, 119,172-175.

40. Peeters, K.; Van Leemputte, F.; Fischer, B.; et al. Fructose-1,6-bisphosphate couples glycolytic flux to activation of Ras Nature commun. 2017, 8, DOI: 10.1038/s41467-017-01019-z.

41. Galluzzi, L.; Kroemer, G. Secondary necrosis: accidental no more Trends Cancer 2017, 3 (1), 1-2.

42. Marshall, M.S.; Gibbs,J.B.; Scolnik,E.M.; Sigal, I.S. Regulatory function of the Saccharomyces cerevisiae RAS C-Terminus Mol. Cell. Biol. 1987, 7, 2309-2315.

43. Hurwitz, N.; Segal,M.; Marbach,I.; Levitzki,A. Differential Activation of yeast adenylyl cycles by Ras1 and Ras2 depends on the conserved N terminus Proc. Natl. Acad. Sci.USA 1995, 92, 11009-11013.

44. Breviario, D.; Hinnebusch, A.G.; Dhar,R. Multiple regulatory mechanisms control the expression of the RAS1 and RAS2 genes of Saccharomyces cervisiae EMBO J1988, 7, 1805-1813.

45. Ghaemmaghami, S., et al. Global analysis of protein expression in yeast Nature 2003, 425, 737-741.

46. Sun, J.; Kale,S.P.; Childness,A.M.; Pinswasdi,C.; Jazwinski,S.M. Divergent roles of RAS1 and RAS2 in yeast longevity J. Biol. Chem. 1994, 269, 18638-18645.

47. Shama, S.; Kirchman,P.A.; Jiang,J.C.; Jazwinski S.M. Role of RAS2 in recovery from chronic stress: Effect on yeast life span Exp Cell Res. 1998, 245, 368-378.

48. Colombo,S. et al. Involvement of distinct G-proteins, Gpa2 and Ras, in glucose- and intracellular acidificationinduced cAMP signalling in the yeast Saccharomyces cerevisiae. EMBO J. 1998, 17, 3326-3341. 
bioRxiv preprint doi: https://doi.org/10.1101/2021.10.13.464237; this version posted October 13, 2021. The copyright holder for this preprint (which was not certified by peer review) is the author/funder. All rights reserved. No reuse allowed without permission.

\section{Supplementary material}

\section{Supplementary Table I}

Primers used in this study

\begin{tabular}{|c|c|c|}
\hline Name & Sequence $\left(5^{\prime} \rightarrow 3^{\prime}\right)$ & Used for \\
\hline ras2delta-URA3-FW & $\begin{array}{l}\text { TAAAAAAACCAAGTTAACCGTTTTCGAATTGAAAGGAGATATACAGAAA } \\
\text { AAAAAGGCAGAACATATCCATCGCG }\end{array}$ & $\operatorname{ras} 2 \Delta$ \\
\hline ras2delta-URA3-RW & $\begin{array}{l}\text { AACCATATGATATTGCCCAAAGTTTCCAATGTTAACAAGAATGAAACGG } \\
\text { CAACGACAGGAGCACGATCA }\end{array}$ & $\operatorname{ras} 2 \Delta$ \\
\hline cnt-ras2delta-FW & CGTTGTCTTCTCTTATCGCC & deletion validation \\
\hline cnt-ras2delta-RW & GATGACTCTCTGCAATGTCC & deletion validation \\
\hline
\end{tabular}

in black: FW nucleotides -1-54 upstream the RAS2 ATG start codon; RW nucleotides $+138+191$ downstream the RAS2 TAA stop codon

in red: URA3 marker Ycp50
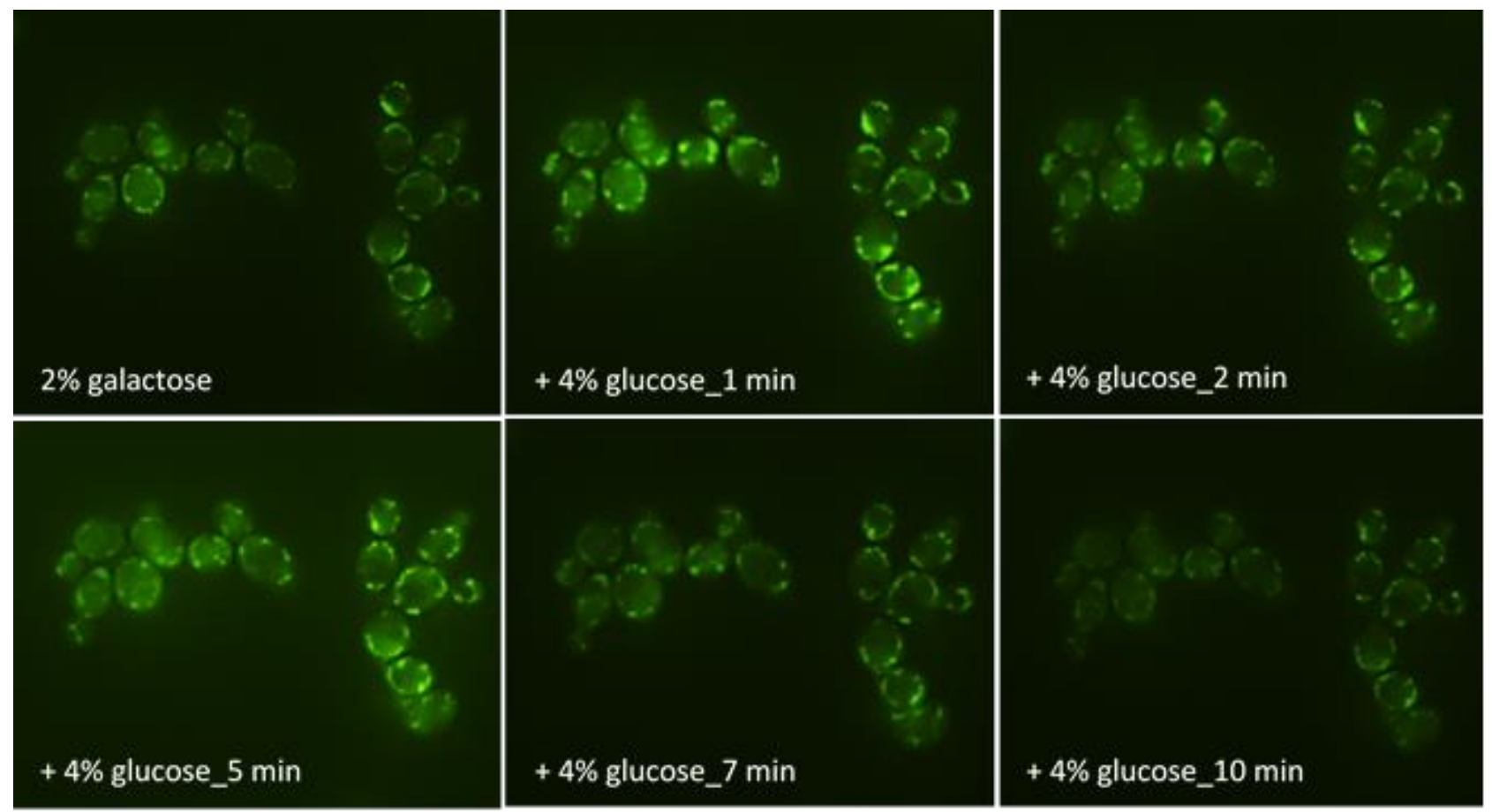

\section{Supplementary Figure 1}

Localization of active Ras proteins in the tps1 $\Delta$ strain before and after addition of glucose to galactose growing cells. Cells expressing the eGFP-RBD3 probe and growing in $2 \%$ galactose medium were seeded on concanavalin A-coated cover glass and mounted on top of a Thoma chamber (see Materials and Methods for details). Images were acquired before and after addition of glucose with a Nikon Eclipse 90i microscope equipped with a 60X oil immersion objective and with the same setting of exposure time and imaging (4s, 1.2 gain). 
A
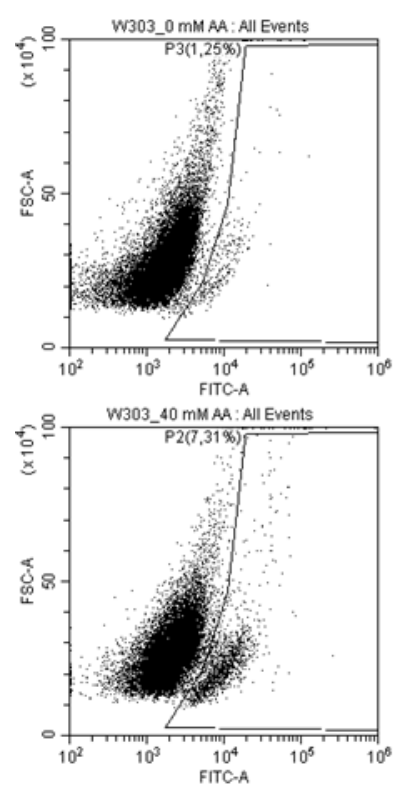
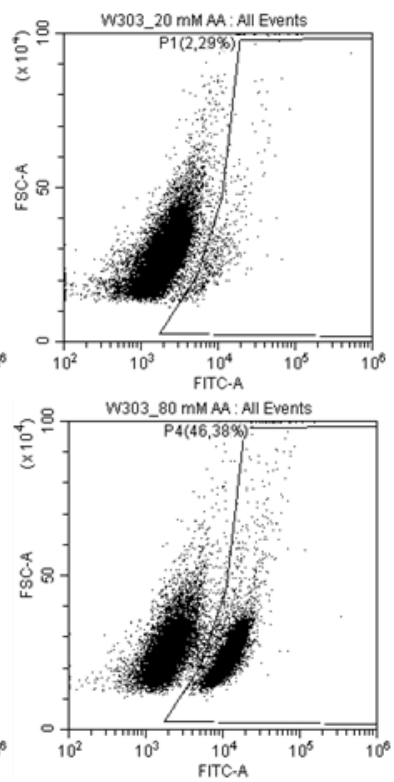

B
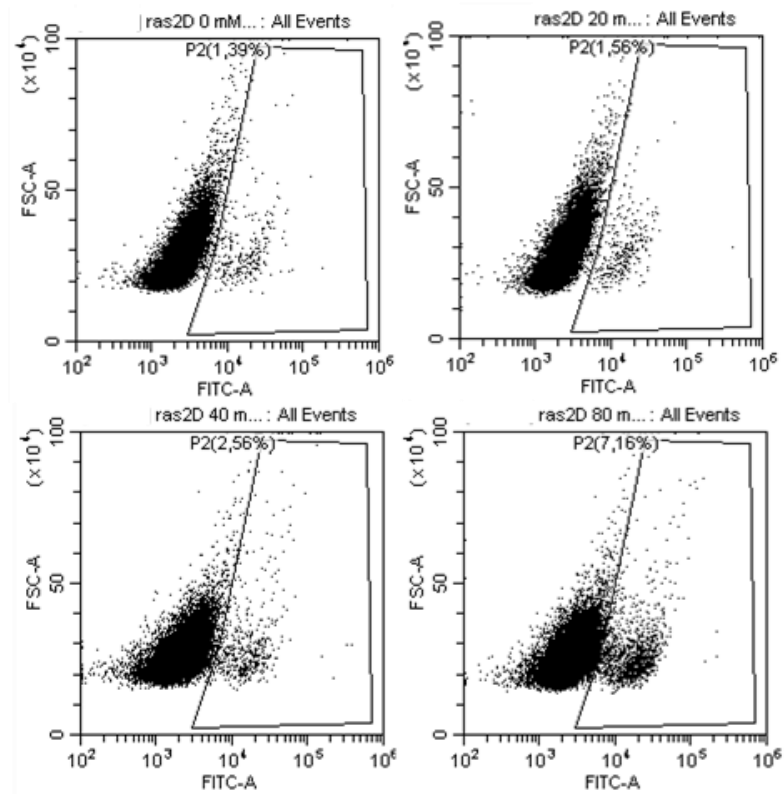

\section{Supplementary Figure 2}

Example of cytofluorimetric analysis after Dihydrorhodamine 123 (DHR123) staining on W303-1A and ras $2 \Delta$ cells. W303-1A (A) and ras2 $\Delta$ (B) exponentially growing cells, untreated or treated with either 20,40 or $80 \mathrm{mM}$ acetic acid for $200 \mathrm{~min}$ at $30^{\circ} \mathrm{C}$. On the $\mathrm{x}$-axis the fluorescence (FITC-A) is shown, while on the y-axis the forward scatter (FSCA) is shown.

A
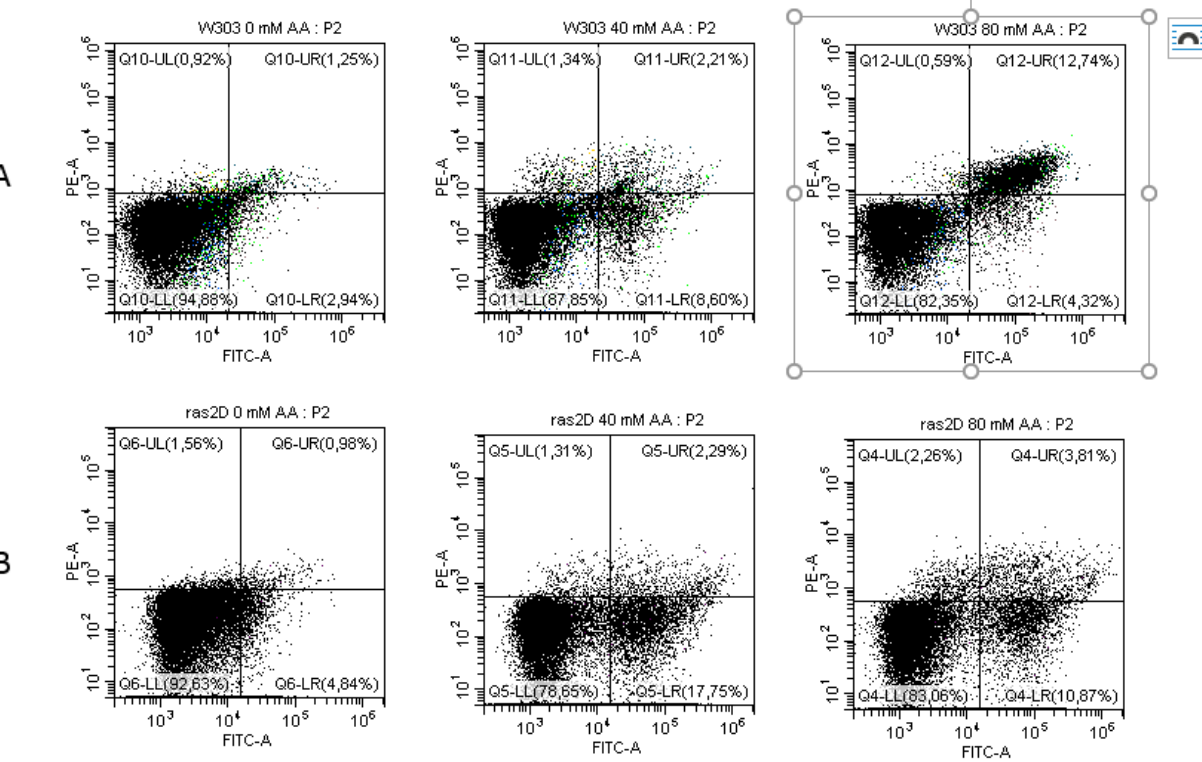

\section{Supplementary Figure 3}

Example of cytofluorimetric analysis after FITC-coupled annexin V and PI staining on W303-1A and ras $2 \Delta$ cells. W303-1A (A) and ras2 $\Delta$ (B) cells growing exponentially on glucose medium were treated with either 40 or $80 \mathrm{mM}$ acetic acid for $200 \mathrm{~min}$ at $30^{\circ} \mathrm{C}$, before being processed for determination of phosphatidylserine externalization and membrane integrity. Cells in the lower left (LL) quadrant of each cell distribution are annexinV-/PI- and represent 
the live population. Cells in the lower right (LR) quadrant are annexinV+/PI- and represent the early apoptotic population. Cells in the upper right (UR) quadrant are annexinV+/PI+ and represent the late apoptotic/secondary necrotic population. Cells in the upper left (UL) quadrant are annexinV-/PI+ and represent the primary necrotic population. 